\title{
Unidad de enfermedades autoinmunes: experiencia de un centro
}

\author{
Autoimmune Diseases Unit: experience of a center \\ Unidade de doenças autoimunes: experiência de um centro
}

Mariana Cruz

ORCID: 0000-0001-7465-4072 Residente Medicina Interna. UdelaR.

Diego Graña

ORCID: 0000-0001-8979-4692 Internista. Profesor Adjunto Clínica

Médica. UdelaR

Dr. Javier Gaudiano

ORCID: 0000-0003-3824-4695

Internista. Asistente Clínica Médica.

UdelaR

Dra. Leticia Pérez ORCID: 0000-0002-0177-2307 Residente Medicina Interna. UdelaR.

Resumen: Introducción: Las enfermedades autoinmunes sistémicas son un grupo de enfermedades de baja prevalencia, cuya patogenia está basada en la pérdida de la auto-tolerancia. A pesar de su baja incidencia y prevalencia, la profundización en el estudio y conocimiento de estas enfermedades ha permitido importantes avances diagnósticos y terapéuticos en los últimos años, constituyendo un desafío en la práctica clínica. Objetivo: determinar la prevalencia y características clínico-humorales de estas patologías en la Policlínica de Enfermedades Autoinmunes del Hospital Pasteur, en el periodo 2016 -2019. Resultados: El total de población fue de 62 pacientes., 55/62 (88\%) son mujeres. La más prevalente fue Lupus Eritematoso Sistémico, representando un $32 \%$ de esta población (20/62), la artritis reumatoide en un $21 \%(13 / 62)$, la esclerosis sistémica en un $8 \%$ (5/62), vasculitis sistémica 8\% (5/62), Overlap 6\% (4/62), Bechet $5 \%$ (3/62). Conclusiones: Se destaca un claro predominio del lupus y de la artritis reumatoide como enfermedades más prevalentes, así como un incremento mantenido de otras patologías menos habituales. Es de suma importancia fomentar el desarrollo de unidades especializadas en estas enfermedades para poder mejorar y protocolizar el manejo de estos pacientes.

Palabras clave: enfermedades autoinmunes sistémicas, perfil clínico, unidad de enfermedades autoinmunes.

Abstract: Introduction: Systemic Autoimmune Diseases are a group of low-prevalence diseases whose pathogeny is based on the loss of self-tolerance. Despite their low incidence and prevalence, deepening the study and knowledge of these diseases has enabled significant diagnostic and therapeutic advances in recent years, constituting a challenge in clinical practice. Objective: to determine the prevalence and clinical-humoral characteristics of these pathologies in the Polyclinic of Autoimmune Diseases of Pasteur Hospital, in the period 2016 -2019. Results: The total population was 62 patients., 55/62 (88\%) were women. Most prevalent was Systemic Lupus Erythematosus (SLE), representing $32 \%$ of this population (20/62), Rheumatoid Arthritis by $21 \%(13 / 62)$, Systemic Sclerosis by $8 \%(5 / 62)$, Systemic vasculitis $8 \%(5 / 62)$, Overlap syndrome $6 \%(4 / 62)$, Bechet $5 \%$ (3/62). Conclusions: Lupus and rheumatoid arthritis as more prevalent diseases, as well as a maintained increase in other less common diseases. It is very important to promote the development of specialized units in these área, in order to improve and protocol the management of these patients.

Key words: systemic autoimmune diseases, clinical profile, autoimmune diseases unit

Resumo. Introdução: As doenças autoimunes sistêmicas são um grupo de doenças de baixa prevalência, cuja patogênese se baseia na perda de autotolerância. Apesar de sua baixa incidência e prevalência, o aprofundamento no estudo e conhecimento dessas doenças permitiu importantes avanços diagnósticos e terapêuticos nos últimos anos, constituindo um desafio na prática clínica. Objetivo: determinar a prevalência e as características clínico-humorais dessas patologias no Hospital Pasteur Polyclinic of Autoimmune Diseases, no período 2016-2019. Resultados: a população total foi de 62 pacientes, 55/62 (88\%) são mulheres. O mais prevalente foi o lúpus eritematoso sistêmico (LES), representando $32 \%$ dessa população (20/62), artrite reumatoide em $21 \%$ (13/62), esclerose sistêmica em $8 \%$ (5 / 62), vasculite sistêmica $8 \%$ 
(5/62), sobreposição de 6\% (4/62), Bechet 5\% (3/62). Conclusões: Uma clara predominância de lúpus e artrite reumatoide destaca-se como as doenças mais prevalentes, bem como um aumento sustentado de outras patologias menos comuns. É de extrema importância promover o desenvolvimento de unidades especializadas nessas doenças, a fim de melhorar e protocolar o manejo desses pacientes.

Palavras-chave: doenças auto-imunes sistêmicas, perfil clinic, unidade de doenças autoimunes.

Recibido: 11/03/2020 Aceptado: 30/05/2020

Clínica Médica 2. Prof. Dra. Mabel Goñi. Hospital Pasteur. Facultad de Medicina. Universidad de la República (UdelaR) Montevideo, Uruguay.

Correspondencia: E-mail: diegograna@adinet.com.uy 


\section{Introducción}

Las enfermedades autoinmunes sistémicas (EAS) son causadas por una alteración en la respuesta adaptativa específica basada en la pérdida de la auto regulación, clínicamente caracterizadas por la afección de múltiples órganos, siendo su diagnóstico y tratamiento un desafío en la práctica clínica ${ }^{(1-3)}$.

Estas enfermedades afectan alrededor de 3 a $5 \%$ de los seres humanos, especialmente a las mujeres ${ }^{(1,2)}$. A pesar de su baja incidencia y prevalencia, la profundización en el estudio y conocimiento de estas enfermedades ha permitido importantes avances terapéuticos en los últimos años, teniendo un cambio sustancial en el pronóstico de las mismas, y en la calidad de vida de los pacientes. Por ejemplo, la incidencia de lupus eritematoso sistémico (LES), enfermedad prototipo de las EAS, se ha triplicado en los últimos 40 años, vinculado probablemente a una búsqueda más exhaustiva con herramientas más específicas y a tratamientos que han prolongado la sobrevida ${ }^{(4)}$.

Se han creado unidades de EAS a nivel mundial y regional, lo que ha permitido el surgimiento de grupos de estudio y trabajo, destinados no solo a la labor asistencial sino también a la investigación, con un beneficio claro para la comunidad científica y sobre todo para los pacientes.

El objetivo del presente trabajo es reportar las características clínicas y humorales de los pacientes que se asisten en la Policlínica de Enfermedades Autoinmunes del Hospital Pasteur, en el periodo comprendido entre 2016 al 2019.

\section{Materiales y métodos}

Estudio observacional, descriptivo de corte transversal, retrospectivo. Se analizaron las pacientes asistidos por diferentes enfermedades autoinmunes, entre 1 de enero de 2016 hasta el 31 de diciembre de 2019.

Criterios inclusión: todos los pacientes asistidos en la policlínica mayores de 15 años, que dieron el consentimiento oral para el estudio. Criterios exclusión: aquellos casos con déficit de datos o que en la evolución se realizó exclusión de EAS.

Se revisaron las historias clínicas de los pacientes, utilizando un formulario pre-codificado para cada una de las enfermedades. La base de datos fue realizada en Microsoft Excel 2016.

Las variables estudiadas fueron: edad, sexo, perfil clínico y de anticuerpos (anticuerpos anti nucleares, anticuerpos anti antígeno extraíble del núcleo, anti DNA, anti fosfolipídicos, anticuerpos anti citoplasma del neutrófilo, factor reumatoideo, anticuerpos anti péptido citrulinado) y tratamiento recibido. 


\section{Resultados}

El presente trabajo es sobre 62 pacientes; con predominio sexo femenino 55/62 (88\%), siendo la edad media de 52 años y la mayor procedencia de Montevideo 51/62 (82 \%).

En la gráfica 1 se presentan las EAS asistidas.

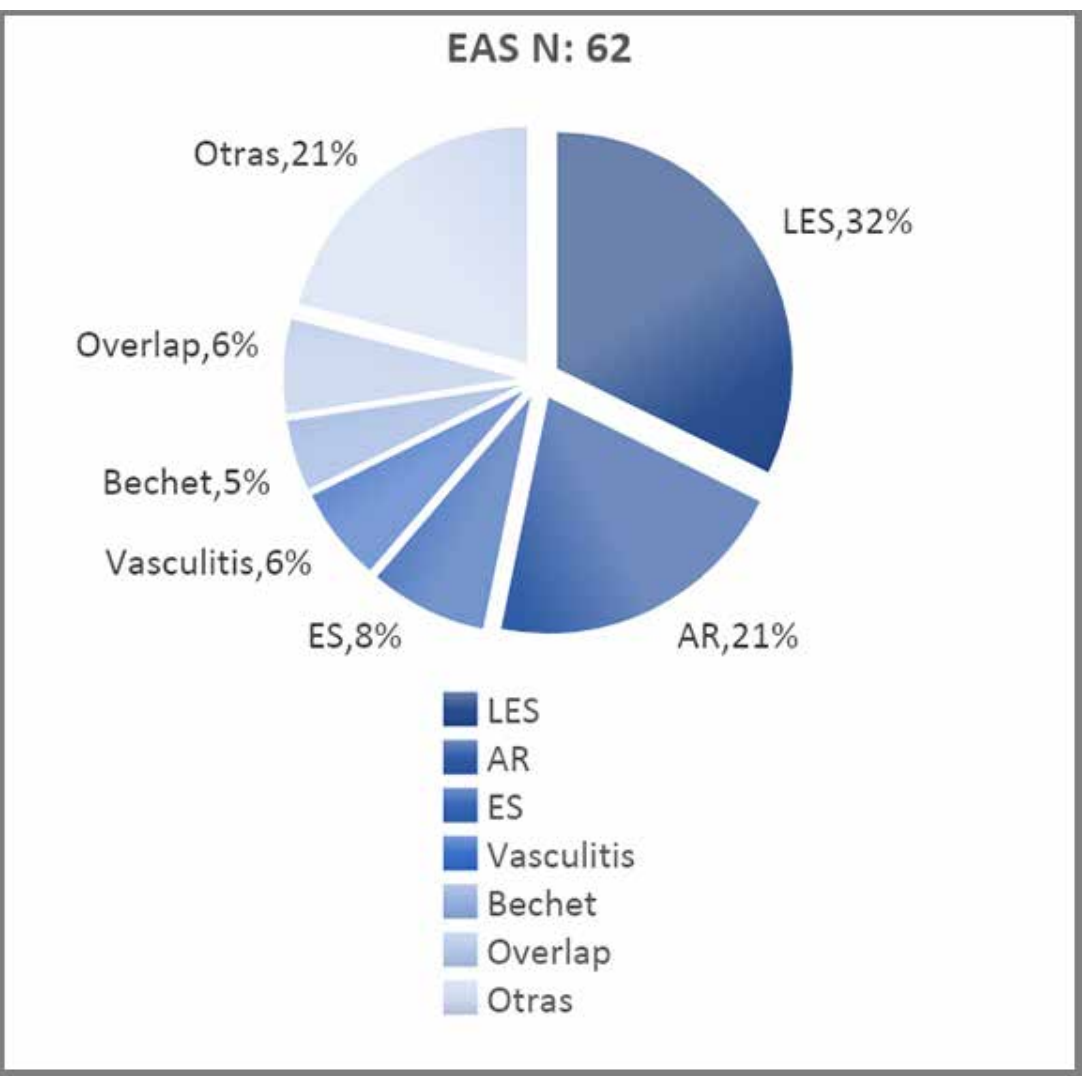

LES (lupus eritematoso sistémico) AR (artritis reumatoidea) ES (esclerosis sistémica)

En el grupo otras (13/62 - 21\%) encontramos casos de Polimiositis, Sarcoidosis pulmonar, Enfermedad mixta del tejido conectivo, Sjogren primario, raynauld primario.

LES (20/62 - 32\%). 90\% son mujeres, la edad media es de 48 años.

En cuanto al compromiso que presentaban, 14/20 era cutáneo, 11/20 articular, 8/20 renal, $7 / 20$ hematológica, 1/20 cardiovascular y 1/20 neurológica.

En la tabla 1 se presentan los auto-anticuerpos encontrados en este subgrupo de pacientes.

\begin{tabular}{|l|l|}
\hline ANA & 17: Positivo (título $\geq 1 / 160)$ \\
\hline & 3: Negativo \\
\hline ENA & 14: Positivo (más frecuente Anti Smith) \\
\hline & 6: Negativo \\
\hline Anti DNA & 8: Positivo \\
\hline & 12: Negativo \\
\hline Complemento & 7: Consumo \\
\hline & 13: Sin consumo \\
\hline
\end{tabular}

El cuanto al tratamiento, el total de los pacientes están con hidroxicloroquina, 9/20 estuvo en algún momento con azatioprina, $7 / 20$ con micofenolato sódico, 3 recibieron ciclofosfamida y sólo 1 paciente recibió rituximab. 
AR (13/62 - 21\%). 92\% son mujeres, la edad media es de 58 años.

Un total de 8 pacientes se presentó como AR aislada, el restante presentó alguna otra manifestación sistémica, predominando dentro de éstas, la vasculitis. Cuatro pacientes presentaron Sjogren secundario.

En cuanto al perfil de anticuerpos; 10/13 pacientes contaron con factor reumatoideo (FR) positivo, y 12/13 con anticuerpos anti péptido citrulinado cíclico (Anti- CCP) positivos. Un paciente es portador de una AR seronegativa.

9/13 pacientes presentaba alteraciones radiológicas características de esta afección como la desmineralización en banda y yuxta articular, geodas, pinzamientos y desviación cubital.

Cuatro pacientes se encuentran en etapa secuelar tanto clínica como radiológica con manifestaciones como la subluxación de las metacarpofalangicas y la desviación cubital, así como imposibilidad de realizar la pinza digital.

En cuanto al tratamiento, el $100 \%$ de los pacientes se encuentran con fármacos modificadores de la enfermedad (FAME). En la gráfica 2 se presentan los mismos.

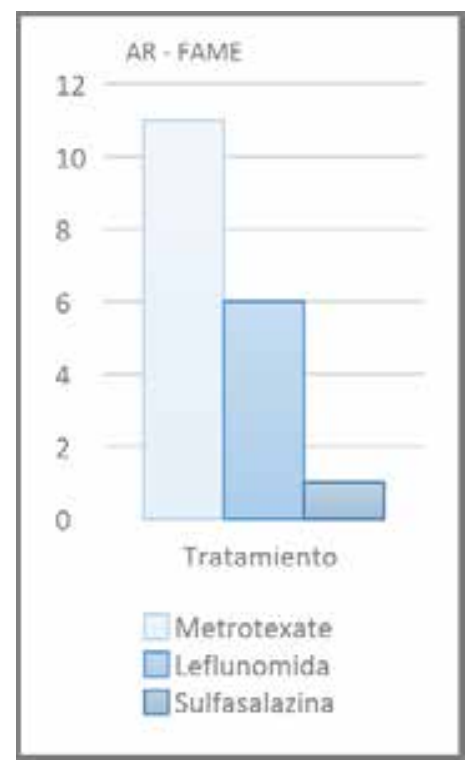

Esclerosis sistémica (5/62 - 8\%), todas mujeres con una edad media de 59 años.

Según la clasificación de la ES, 3 corresponden a difusa, 1 a limitada y 1 a ES sin esclerodermia.

El análisis del perfil clínico de estas pacientes mostró que todas presentan raynauld; 4 compromiso cutáneo; 3 enfermedad por reflejo gastroesofágico (ERGE); 3 afectación pulmonar y 1 paciente presento compromiso articular. De las pacientes con afectación pulmonar, el compromiso intersticial fue su forma de presentación.

Perfil de anticuerpos: todas las pacientes presentaron ANA positivo, 2/5 Anti centrómero positivo y $1 / 5$ Anti-SCL 70 positivo.

El total de las pacientes se encuentran bajo tratamiento para la ERGE y raynauld (calcio antagonistas y sildenafil). Además 1/5 requirió hidroxicloquina 1 y 2/5 micofenolato de mofetilo.

Vasculitis sistémica $(5 / 62-8 \%)$, siendo 3 de sexo femenino.

Los anticuerpos anti citoplasma de neutrófilo (ANCA) tanto dirigidos contra la proteinasa 3 ( PR3) como contra la Mieloperoxidasa ( MPO) fueron en todos los casos negativos. En 2 de los pacientes se estableció el diagnostico a través de biopsia cutánea, resultando las mismas positivas para vasculitis leucocitoclástica. Una sola paciente presento compromiso cutáneo y renal, en los restantes el compromiso fue exclusivamente cutáneo. 
Overlap (4/62 - 6\%), siendo 3 de sexo femenino.

Dos de estos pacientes tienen AR - LES, un paciente LES - enfermedad mixta del tejido conectivo, y el ultimo esclerosis sistémica - polimiositis.

Enfermedad de Bechet (3/62 - 5\%), siendo dos de sexo masculino.

Los tres casos tienen compromiso cutáneo exclusivo, a todos los pacientes se realizó test de patergia, de los cuales dos resultaron positivos. Los tres pacientes se encuentran bajo tratamiento con colchicina.

Contamos con 3 pacientes con Raynauld primario, dos de los cuales corresponden al sexo femenino. Todos ellos se encuentran bajo tratamiento con calcio antagonistas.

\section{Discusión}

EI LES es el prototipo de enfermedad autoinmune sistémica, con una expresión clínica muy heterogénea. Siendo la de mayor prevalencia en nuestra población y predominando el sexo femenino (90\%). En las últimas décadas ha aumentado la incidencia de LES, en parte debido a la mayor detección de casos en estadios iniciales de la enfermedad y a una búsqueda más exhaustiva de la misma. Actualmente tiene una prevalencia estimada de 20-150/100.000 habitantes ${ }^{(5,6)}$.

En nuestro estudio la mayoría de los pacientes presentan compromiso cutáneo y articular, 8 pacientes presentaron afección renal, dentro de los cuales 6 contaban con biopsia renal, siendo en todos los casos compatibles con nefropatía lúpica tipo IV. En Uruguay según los datos del Registro Uruguayo de Glomerulopatías, la glomerulonefritis lúpica es hallada en $32-42 \%$ del total de biopsias reportadas ${ }^{(4)}$. La totalidad de los pacientes biopsiados con nefropatía lúpica presentaban anticuerpos anti-DNA a títulos elevados. Estos anticuerpos preceden muchas veces al desarrollo de la nefritis lúpica clínica, como muestra la literatura internacional ${ }^{(4,5,7,8)}$. El $54 \%(11 / 20)$ presentaron este anticuerpo positivo.

Los hombres con lupus tienen con mayor frecuencia afectación renal, serosa y enfermedad cardiovascular respecto a las mujeres ${ }^{(5,9,10)}$. Los únicos dos hombres de nuestra población desarrollaron enfermedad renal, con compromiso cutáneo, ambos de muy difícil manejo, comportándose uno de ellos como un lupus cutáneo refractario a tratamiento inmunosupresor, requiriendo finalmente rituximab.

El $85 \%$ de los pacientes presentaron ANA positivo a títulos mayores a $1 / 160,82 \%$ presentaron ENA positivo, siendo el más frecuente Anti Smith, anticuerpo altamente especifico de esta enfermedad ${ }^{(4,5)}$.

Respecto al tratamiento; los antipalúdicos (hidroxicloroquina) constituye el tratamiento base ${ }^{(3-}$ 5,11,12). En personas con LES, su uso disminuye la mortalidad por cualquier causa, daño orgánico irreversible, infecciones graves, trombosis arteriales y venosas y síndrome metabólico ${ }^{(4,5,11,13)}$. Es por ello que la totalidad de los pacientes con LES asistidos en nuestra unidad se encuentran bajo tratamiento con hidroxicloroquina.

La mayoría de los pacientes recibe o recibió en algún momento tratamiento inmunosupresor, ya sea para inducción o mantenimiento de la enfermedad. Los fármacos utilizados fueron azatioprina, micofenolato de mofetil, ciclofosfamida y rituximab.

El micofenolato hoy en día es el tratamiento de primera línea para el manejo de la nefropatía lúpica y es utilizado en casos de lupus cutáneo refractario, serositis y en algún caso de compromiso neurológico ${ }^{(14)}$. Para la enfermedad renal, tiene la misma eficacia que la ciclofosfamida, con un menor perfil de toxicidad, se ha demostrado que el micofenolato es equivalente al uso de bolos de ciclofosfamida como terapia de inducción en la nefropatía lúpica $\left.{ }^{(5,6,11,15,16}\right)$. Solo un paciente recibió tratamiento biológico (rituximab), tratándose de un caso de lupus cutáneo refractario.

Las guías actuales recomiendan el uso de rituximab en aquellos pacientes con refractariedad al tratamiento de primera línea y con afectación grave (renal, neurológico, etc. ${ }^{(5-7,12)}$.

La artritis reumatoide es una enfermedad inflamatoria crónica sistémica caracterizada por destrucción articular que se presenta mayormente en edades tempranas, su mayor incidencia se da entre los 35 a 45 años y afecta entre el 0,03 y el $1,5 \%$ de la población mundial ${ }^{(17,18)}$.

Contamos con pocos pacientes portadores de AR, en comparación con registros nacionales e internacionales, probablemente se encuentre explicado por la presencia de otras unidades especializadas en la captación de pacientes con AR en nuestro hospital. 
Al igual que el resto de las EAS, predomina en mujeres, pero a diferencia de lo que sucede en otros centros nacionales y regionales, la mayoría de nuestras pacientes son añosas, vinculado probablemente a una captación tardía, siendo su diagnóstico realizado una vez que la enfermedad se encuentra en una etapa secuelar. Solo en 3 de las 13 pacientes se realizó el diagnostico en una etapa precoz. Creemos que esto puede incidir en la baja tasa de pacientes en tratamiento con fármacos biológicos dentro de esta enfermedad.

Un hecho interesante en nuestra población es que el compromiso extraarticular no se caracterizó por el compromiso pulmonar, como habitualmente esta descrito en la literatura, sino que, se manifestó como vasculitis cutánea asociada (3 pacientes), compromiso poco frecuente en esta enfermedad ${ }^{(17-19)}$.

Como enfermedad autoinmune asociada el síndrome de Sjögren se presentó en 4 de ellas. Su búsqueda sistemática permite detectarlo en el 10-30\% de los pacientes según las series ${ }^{(18)}$. Esta enfermedad cuando es secundaria a la AR suele tener un curso más benigno que el síndrome de Sjögren primario, sobre todo con menos compromiso parotídeo y manifestaciones extra glandulares, sin modificarse el riesgo de evolucionar al linfoma ${ }^{(17,18)}$.

En cuanto al perfil inmunológico tenemos una alta prevalencia de AR seropositivas, 10 para el FR y 12 para los Anti-CCP. Se ha demostrado cierta correlación entre la positividad del FR y la progresión radiológica de la AR. Además, las AR graves con signos extraarticulares son casi siempre intensamente seropositivas, como sucedió en las pacientes de nuestras series ${ }^{(18,19)}$.

Solo una paciente se presentó con AR seronegativa, en nuestra serie. Estando esto en línea con lo que refiere la bibliografía internacional, donde únicamente un 15-20\% de las AR están bajo este rotulo ${ }^{(18,19)}$

El tratamiento farmacológico de la AR se divide en sintomático (analgésicos, antiinflamatorios no esteroideos [AINE], corticoides) y los fármacos modificadores de la enfermedad, como metrotexato (MTX), leflunomida, sulfasalazina e hidroxicloroquina ${ }^{(18,20)}$. En nuestra unidad 13 pacientes se encuentran bajo metrotexato, de los cuales 6 asocian leflunomida y 1 sulfasalazina.

La eficacia clínica del MTX ha sido demostrada en numerosos estudios. El porcentaje de pacientes que responden al MTX es de un 50-60\%. La leflunomida ha demostrado una eficacia y una tolerabilidad comparables a las del MTX ${ }^{(18,20)}$.

Hoy en la actualidad, el uso de biológicos en esta enfermedad se ha incrementado en forma considerable, reduciendo los plazos para implementar su uso. Los anti TNF constituyen el Gold standard (adalimumab; etarnercept; infliximab), utilizándose también otros fármacos como el rituximab y el tocilizumab ${ }^{(21,22)}$. Así mismo, existen otras dianas terapéuticas en pleno desarrollo.

La esclerosis sistémica es una enfermedad infrecuente, en nuestra policlínica representa el $8 \%(5 / 62)$ de la población y a nivel mundial su prevalencia es de 0,6-1.22 casos anuales por millón de habitantes ${ }^{(23)}$. Debido a la diversidad de las formas clínicas, subclínicas y los síndromes de superposición con otras enfermedades del tejido conectivo existe una subestimación de la incidencia y de la prevalencia reales de la enfermedad ${ }^{(23,24)}$.

Según la extensión del compromiso cutáneo se puede clasificar a la enfermedad en: ES difusa, ES limitada y ES sin esclerodermia, siendo la más frecuente la forma difusa, como vimos en nuestra serie, 3 de las 5 pacientes se presentaron de esta manera ${ }^{(23,25)}$.

El síntoma precoz y casi constante de la enfermedad es el síndrome de Raynaud, en nuestro estudio todos los pacientes con ES presentaron Raynauld ${ }^{(23,24,26)}$. A nivel mundial se observa en el $96-98 \%$ de los casos. El tratamiento farmacológico en base a los antagonistas del calcio ha demostrado en varios ensayos controlados aleatorizado su eficacia para reducir la frecuencia y la gravedad del síndrome de Raynaud, por lo que los 5 pacientes con ES se encuentran bajo dicho tratamiento.

La afectación digestiva es frecuente en la ES, se observa en el 75-90\% de los pacientes, en nuestra serie 4 de las 5 pacientes lo presentaron. Puede afectar a todo el tubo digestivo, desde el esófago hasta el recto. Se produce tanto en las formas cutáneas limitadas como en las formas cutáneas difusas ${ }^{(23,24,26)}$.

Las manifestaciones pulmonares son la principal causa de muerte en la ES ${ }^{(23)}$. El compromiso pulmonar más frecuente es la enfermedad pulmonar intersticial difusa (EPID) y la hipertensión pulmonar (HTP) ${ }^{(23)}$. La EPID ocurre con mayor frecuencia en los casos de esclerosis sistémica difusa y la HTP es más frecuente en la forma limitada ${ }^{(23,24,26)}$. De los 5 casos de ES, 3 tienen 
compromiso pulmonar, a forma de EPID. Ninguna de las pacientes presento hipertensión pulmonar. El inmunosupresor utilizado en estos pacientes fue el micofenolato de mofetilo (27).

El análisis de los anticuerpos mostro la presencia de anticuerpos antinucleares en todos los pacientes. Encontramos anticuerpos anti centrómero en 2 pacientes, predominando en la forma limitada. Los anticuerpos anti topoisomerasa I (SLC-70) fueron positivos en un paciente. Estos anticuerpos se asocian con compromiso pulmonar a forma de EPID, y los anti centrómero a la HTP, en nuestro caso de los tres pacientes con EPID 2 presentaron anti centrómero positivo y solo uno SLC-70 positivo.

Respecto a las vasculitis, los cuatro casos que tenemos correspondieron a vasculitis leucocitoclásticas, habiendo realizado biopsia en dos de ellas, caracterizándose por el depósito de IgG. En nuestra serie ninguno de los pacientes mostró la presencia de ANCA.

\section{Conclusiones}

Si bien la población asistida en nuestra unidad ha ido aumentando con el transcurrir de los meses, ésta representa una pequeña parte del espectro total de los pacientes con enfermedades autoinmunes. Creemos importante continuar con el desarrollo de estas unidades de EAS y la interconexión con otros centros para poder contar con volúmenes más importantes de pacientes a la hora de realizar recomendaciones y pautar estrategias terapéuticas.

\section{Bibliografía}

1- Finger DS. Persisten las preguntas Factores ambientales en las enfermedades autoinmunes [Internet]. Salud Pública de México. 2011[acceso: 06/06/2020] (53): 4 Disponible en: http://saludpublica.mx/index.php/spm/rt/printerFriendly/7080/9144

2- Dutz JP, Jacobe HT, Sontheimer RD. Autoanticuerpos encontrados en pacientes con trastornos autoinmunitarios del tejido conjuntivo. EN: Bolognia J, Schaffer J, Cerroni L. ed. Dermatología reumatológica. Barcelona: Elsevier 2018 p. 649-661.

3- Cruz DPD, Khamashta MA, Hughes GRV. Systemic lupus erythematosus. Lancet. 2007 Feb 17;369(9561):587-96.

4- Lazaro E, Richez C, Seneschal J. Lupus eritematoso sistémico. EMC. 2015; 48(1): 1-17.

5- Trujillo-Martín MM, Rúa-Figueroa Fernández de Larrinoac I, Ruíz-Irastorza G, Pego-Reigosa JM, Sabio Sánchez JM, Serrano-Aguilar P. Guía de práctica clínica para el lupus eritematoso sistémico: recomendaciones para el abordaje clínico general. Med Clin (Barc). 2016. http://dx.doi.org/10.1016/j. medcli.2016.01.013

6- Durcan L, Dwyer TO, Petri M. Management Strategies and Future Directions for Systemic Lupus Erythematosus in Adults. Lancet. 2019 Jun 8;393(10188):2332-2343. doi: 10.1016/S01406736(19)30237-5

7- Crow MK. Systemic Lupus Erythematosus. In: Goldman-Cecil. Medicine. 26 th ed. Set. Elsevier; 2018. vol.2. p. 1725-1734.

8- Kokuina E, del Toro M, Gutierrez A, Ortiz Alfredo, Sanchez Y, Perez D. Anticuerpos antinucleares específicos y afectaciones orgánicas en 180 pacientes con lupus eritematoso sistémico. Rev Cuba Reumatol. 2015; 17(2): 104-11.

9- Romani F, Atencia F, Cuadra J, Canelo C. Lupus eritematoso sistémico, en un paciente varón : a propósito de un caso. An Fac med. Perú 2008;69(1):37-41.

10- Pinto L, Velazquez J, Marquez J. Subgrupos de Lupus Eritematoso Sistémico. Rev Colomb Reumatol. 2008; 15(4):291-8.

11- Dörner T, Furie R. Review Novel paradigms in systemic lupus erythematosus. Lancet. 2019; 393 (4):2344-58.

12- Fanouriakis A, Kostopoulou M, Alunno A, Aringer M, Bajema I, Boletis JN, et al. 2019 Update of the EULAR recommendations for the management of systemic lupus erythematosus. Ann Rheum Dis 2019;78:736-745.13.

13- Danza Á, Graña D, Goñi M, Vargas A, Ruiz-irastorza G. Hidroxicloroquina en el tratamiento de las enfermedades autoinmunes sistémicas. Rev Med Chile. 2016; 144(2): 232-40.

14- Graña D, Silveira G, Goñi M, Danza A. Lupus neuropsiquiátrico . A propósito de tres casos y revisión de la literatura. Rev Urug Med Inter. 2020; 3(1): 33-40. 
Rev. urug. med. interna.

15- Bomback A, Agati. Systemic Lupus Erythematosus and the Kidney. En: Gilbert SJ, Weiner DE. National Kidney Foundation Primer on Kidney Diseases. $7^{\text {a }}$ ed. Philadelphia: Saunders: 2014. p. 245-251.

16- Appel G, Contreras G, Dooley MA, Ginzler EM, Isenberg D, Jayne D, et al. Mycophenolate Mofetil versus Cyclophosphamide for Induction Treatment of Lupus Nephritis. J Am Soc Nephrol. 2009 May;20(5):1103-12. doi: 10.1681/ASN.2008101028 17

17- Mcinnes I, Dell JRO. Rheumatoid Arthritis. In: Goldman-Cecil. Medicine. 26 th. Philadelphia: Elsevier: 2018. vol.2. p. $1709-1718$.

18- Combe B, Lukas C, Morel J. Artritis reumatoide del adulto : epidemiología, clínica y diagnóstico. EMC. 2015; 48(4):1-17.

19- Smolen JS, Aletaha D, Mcinnes IB. Rheumatoid arthritis. Lancet 2016; 388(6):2023-38.

20- Dell JRO. Tratamiento de la artritis reumatoide. Kelley y Firestein. En: Tratado de reumatología. 10ed. 2018. p. $1187-1212$.

21- Pecoraro V, Santis E De, Melegari A, Trenti T. The Impact of Immunogenicity of TNF囚 Inhibitors in Autoimmune Inflammatory Disease. A Systematic Review and Meta-Analysis. Autoimmun Rev. 2017 Jun;16(6):564-575. doi: 10.1016/j.autrev.2017.04.002

22- Tutuncu Z, Kavanaugh A. Tratamientos anticitocinas. En: Kelley y Firestein. Tratado de reumatología. $10^{\mathrm{a}}$ ed. 2018. p. 999-1019.

23- Varga J, Wigley FM. Esclerodermia-esclerosis generalizada. En: Inmunologia clinica. $5^{a}$ ed. 2017. p. 743-755.

24- Puzenat E, Aubin F, Humbert P. Esclerodermia sistémica. EMC - Dermatol. 2010;44(2):1-15.

25- Elhai M, Avouac J, Kahan A, Allanore Y. Esclerodermia sistémica. EMC - Aparato Locomotor. 2015;48(3):1-15.

26- Systemic Sclerosis (Scleroderma). En: Goldman-Cecil. Medicine. 26th. Elsevier; 2018. vol. 2. p. 17341742.

27- Hinshaw M, Lawrence-hylland S. Connective Tissue Disorders. Conn's Current Therapy 2020. Elsevier; 2018. p. 918-921.

\section{Aporte cada autor al trabajo}

Mariana Cruz: 50\%

Diego Graña: 30\%

Javier Gaudiano: 10\%

Leticia Pérez: 10\% 